\title{
Influence of doping on the performance of GaAs/AIGaAs QWIP for long wavelength applications
}

\author{
A. BILLAHA* and M.K. DAS \\ SAP Research Laboratory, Dept. of Electronics Engineering, Indian School of Mines, \\ Dhanbad - 826004, Jharkhand, India
}

\begin{abstract}
Effect of doping and other device parameters on inter sub-band transition in the well, responsivity and dark current of $G a A s / A l_{x} G a_{l-x} A s$ quantum well infrared photodetector $(Q W I P)$ is investigated using theoretical model. $2 X 2$ Hamiltonian method is used to calculate Eigen energy states in this modelling. Results show that peak absorption, responsivity and spectral broadening width increase nonlinearly with increasing doping concentration in the well. Peak absorption coefficient increases with increase in well width also. Moreover, with increase in mole fraction of $\mathrm{Al}$ in $A l_{x} G a_{l_{-x}} A s$ barrier, the inter sub-band absorption is enhanced but, peak wavelength of absorption shifts towards shorter wavelengths. Dark current density depends on both, the doping concentration and applied bias.
\end{abstract}

Keywords: Quantum wells, inter sub-band absorption, doping concentration, responsivity, dark current.

\section{Introduction}

Extensive progresses in the study of inter sub-band absorption in quantum well (QW) structures for detection of wavelength in infrared region has been done over the past few decades [1-6]. Researchers have also shown their interest in the design of photodetector based on QW structure because of its potentiality to use in sophisticated applications like medical science, night vision camera, thermal imaging, defence, etc. [7-8]. Moreover, its performance can be engineered by controlling transport mechanism of photo excited carriers, mechanisms of injection and capture of carriers into the QWs, etc. [2-6]. HgCdTe based quantum well infrared photodetector (QWIP) has already proved its performance in this field but, cadmium is toxic material and so not suitable for environment friendly applications [9-10]. Recently, GaAs-based QWIP has emerged as one of the most promising photodetectors for infrared (IR) imaging application in the Long Wavelength (LW) IR region [11]. Advantages of GaAs-based QWIP include easy wavelength adjustment, high thermal salability and high uniformity which recognized them as high performance detectors for third generation infrared cameras [12]. In addition, GaAs/ $\mathrm{Al}_{\mathrm{x}} \mathrm{Ga}_{1-\mathrm{x}} \mathrm{As}$ based QWIPs can be designed for various applications by controlling $\mathrm{Al}$ composition in AlGaAs layer and by controlling thickness of this layer [13].

Now, doping in the active layer of QWIP plays an important role on the performance of the device. Moreover, wavelength of operation of the QWIP depends on the doping in the active well layer [14]. Thus, choice of optimum

\footnotetext{
*e-mail: rf.billaha@gmail.com
}

doping concentration for enhanced performance of the device is very important. Few works have already been carried out in this context, e.g., Gunapala et al. experimentally investigated the effect of doping on the QWIP performance based on bound-to-continuum transition [15]. But the work has been reported without any theoretical analysis in detail. So, the physics based accurate numerical modelling is required to study the effect of doping in detail on the performance of these devices. In this article, we represent a theoretical model for $\mathrm{GaAs} / \mathrm{Al}_{\mathrm{x}} \mathrm{Ga}_{1-\mathrm{x}}$ As multiple quantum well (MQW) infrared photodetector (IP) considering the effect of doping on the potential in the well and hence on the overall performance of the device. Some best possible values of doping concentrations for enhanced performance are also suggested. Dark current has always an important role on the performance of photodetector. Doping dependent dark current has also been analysed in the present work. This model is applicable for other material systems also like, InGaAs/ $\mathrm{AlGaAs}, \operatorname{InGaAs}(\mathrm{P}) / \mathrm{InP}$ and $\mathrm{Si} / \mathrm{SiGe}$, etc. to understand the effect of doping on the performance of the device. Rest of the paper is organized as follows. In Sect. II, the physics based theoretical model for QWIP is described. Simulation results are discussed in Sect. III and a summary of the work with conclusion is given in Sect. IV.

\section{Theoretical model}

Device structure of GaAs/ $/ \mathrm{Al}_{\mathrm{x}} \mathrm{Ga}_{1-\mathrm{x}} \mathrm{As}$ MQW IP, considered in our analysis, is shown in Fig. 1(a). Alternate layers of GaAs well and $\mathrm{Al}_{\mathrm{x}} \mathrm{Ga}_{1-\mathrm{x}} \mathrm{As}$ barrier on semi-insulating GaAs substrates is considered to form a multiple quantum well structure. GaAs layer is considered to be mainly responsible 


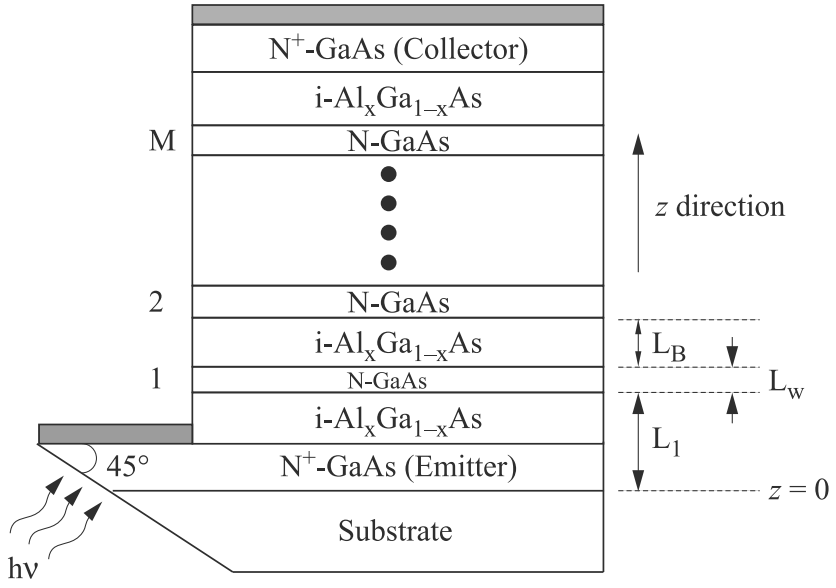

(a)

$$
\left|\begin{array}{c|c|c}
\begin{array}{c}
\text { Region } \\
\mathrm{r}=1
\end{array} & \mathrm{r}=2 & \mathrm{r}=3 \\
\mathrm{Al}_{\mathrm{x}} \mathrm{Ga}_{1-\mathrm{x}} \mathrm{As} & \mathrm{GaAs} & \mathrm{Al}_{\mathrm{x}} \mathrm{Ga}_{1-\mathrm{x}} \mathrm{As}
\end{array}\right|
$$

(b)

Fig. 1. (a) Schematic layer structure of a GaAs/ $\mathrm{Al}_{\mathrm{x}} \mathrm{Ga}_{1-\mathrm{x}} \mathrm{As} \mathrm{MQW}$ infrared photodetector. 1,2. M shows the number of QW. (b) Lateral view of a single QW (barrier-well-barrier) layer structure.

for absorption, i.e., active layer and is doped with Si donors where $\mathrm{Al}_{\mathrm{x}} \mathrm{Ga}_{1-\mathrm{x}} \mathrm{As}$ barrier layer is undoped. Width of the well and barrier are symbolized as $L_{w}$ and $L_{B}$, respectively. Multiple periods of well and barrier are sandwiched between $0.7 \mu \mathrm{m}$ thick GaAs top contact layer (doped with $2 \times 10^{18} \mathrm{~cm}^{-3} \mathrm{Si}$ donors) and $0.5 \mu \mathrm{m}$ thick GaAs bottom contact layer ( $\mathrm{Si}$ doped to $2 \times 10^{18} \mathrm{~cm}^{-3}$ ). Light is assumed to be incident on the detector at an angle $(\phi), 45^{\circ}$ with the growth axis $(z)$ as shown in figure.

One of the important performance parameters of photo detector is its responsivity which gives a measure of sensitivity, as well. Calculation of responsivity needs determination of photocurrent density which in turn requires calculation of the absorption coefficient $(\alpha)$. Absorption coefficient concerning the inter sub band transition in the well is calculated first and, then, the photocurrent density is determined. To determine the absorption spectra, a section (barrier-well-barrier) of the device, as shown in Fig. 1(b), is considered. The electronic structure of the quantum well can be obtained by solving one dimensional (1D) timeindependent Schrödinger equation for the wave function of electron $\psi(z)$ with energy $E$ as given below

$$
-\frac{\hbar}{2 m_{e}^{*}} \frac{\partial^{2} \psi(z)}{\partial z^{2}}+\left[V(z)+V_{H}(z)-q \varepsilon z\right] \psi(z)=E \psi(z) .
$$

Here, $\hbar$ is the reduced Planck constant, $m_{e}^{*}$ is the effective mass of electron, $V(z)$ is the potential due to band offset, $q$ is the electron charge and $\varepsilon$ is the electric field. The
Hartree potential, $V_{H}(z)$ is obtained by solving 1D Poisson equation [16]

$$
\frac{\partial^{2} V_{H}(z)}{\partial z^{2}}=\frac{q}{\varepsilon_{0} k}[N(z)-n(z)],
$$

where, $\varepsilon_{0}$ is the vacuum electric permittivity, $k$ is the relative permittivity of GaAs material, $N$ is the quantum well doping concentration and the electron concentration is $n(z)$ which can be written as

$$
n(z)=\sum_{j} n_{2 D, j}\left|\psi_{j}(z)\right|^{2},
$$

where $j$ stands for different sub-bands and summation is taken over all sub-bands. Following Fermi-Dirac statistics, the $j^{\text {th }}$ sub-band occupation, $n_{2 D, j}$ is given by

$$
n_{2 D, j}=\frac{m_{e}^{*} k_{B} T}{\pi \hbar^{2}} \ln \left[1+e^{\left(E_{F}-E_{j}\right) / k_{B} T}\right] .
$$

where, $E_{F}$ is the Fermi energy, $k_{B}$ is the Boltzmann constant, $T$ is the lattice temperature.

Self-consistent solution of Eq. (1) and Eq. (2) is obtained as follows. Initially, Eq. (1) is solved, to obtain energy Eigen values and the electron wave functions, by assuming some initial value of Hartree potential, $V_{H}(z)$ and by applying the $2 \times 2$ Hamiltonian techniques. In absence of electric field, solution of Eq. (1) can be written as

$$
\psi_{r}(z)=A_{r} e^{i k_{r} z}+B_{r} e^{-i k_{r} z}
$$

where, $A_{r} \& B_{r}$ are two coefficients,

$$
k_{r}=\sqrt{2 m_{r e}^{*}\left(E-V_{r}\right) / \hbar^{2}} \text { for } E>V_{r}
$$

and $k_{r}=i k_{r}=i \sqrt{2 m_{r e}^{*}\left(V_{r}-E\right) / \hbar^{2}}$ for $E<V_{r}$ is the wave number, $r$ is an integer (e.g., 1,2,3...) which signifies the regions as shown in Fig. 1(b). For the region $z<0, r=1$, for region, $0 \leq z \leq L_{w}, r=2$ and for region, $z>L_{w}, r=3$. It is implied by Eq. (4) that, for bound states, both the coefficients, $A_{r} \& B_{r}$ exist within the region of quantum well (QW), i.e., $0 \leq z \leq L_{w}$ and the wave function is continuous at the boundaries of well and barriers. Applying boundary conditions, i.e., continuity of $\psi_{r}(z)$ and $\left[1 / m_{r e}^{*}\right]\left[\partial \psi_{r}(z) / \partial z\right]$ at the boundaries, matrix relationships of $A_{r+1}$ and $B_{r+1}$ with the neighbouring region coefficients $A_{r}$ and $B_{r}$ are obtained. This matrix is further used to calculate Eigen energy states. $A_{r}, B_{r}$ and $k_{r}$ varies from region to region whereas, the wave function, $\psi_{r}$ varies with $z$ even within a region. So, each region is divided into several elemental pieces of equal and small length, $\Delta z$ and $\psi_{r}(z)$ is calculated for all of these elemental lengths. However, the potential is assumed to be constant within a particular region [17]. Very small elemental length is considered in order to minimize the error. However, under the application of electric field, solution of Eq. (1) is different from Eq. (4) and is given by 


$$
\psi_{r}(z)=A_{r} A_{i}\left[\left(z \varepsilon_{r}+l_{r}\right) / \xi_{r}\right]+B_{r} B_{i}\left[\left(z \varepsilon_{r}+l_{r}\right) / \xi_{r}\right],
$$

where $l_{r}=\left(E-V_{r}\right) / q, \zeta_{r}=-\sqrt{\varepsilon_{r}^{2} \hbar^{2} / 2 m_{r e}^{*} q}, A_{i} \& B_{i}$ are the Airy functions of the first and second kind, respectively. Calculated Eigen values and wave functions, after solving Eq. (1), are now used to find the doping dependent Hartree potential, $V_{H}(z)$ in Eq. (2). Initially assumed $V_{H}(z)$ is replaced by this calculated (first modified) value and Eq. (1) is solved again with this modified value of $V_{H}(z)$ to obtain first modified Eigen energies and wave functions. These modified values are used to calculate second modified $V_{H}(z)$ which replaces first modified $V_{H}(z)$ to find solution of Eq. (1) further to obtain second modified values of Eigen energies and wave functions. This cycle is repeated until self-consistent solution for $V_{H}(z)$ and, hence, Eigen energies and wave functions are obtained. Difference between two successively modified values of Eigen energies is used as parameter for convergence.

Absorption coefficient concerning transition between discrete energy levels in the well can be determined by using Fermi's golden rule for a harmonic perturbation [18]. The absorption coefficient $\alpha_{C_{B-B}}(\hbar \omega)$ for bound-to-bound (B-B) transitions can be written as

$\alpha_{C_{B B}}(\hbar \omega)=\frac{q^{2}}{\left(m_{w e}^{*}\right)^{2}} \frac{\hbar^{3}}{\varepsilon_{0} n_{r} c} \frac{N}{\hbar \omega}\left[\mid\left\langle\left.\psi_{f}(z)\left|\frac{\partial}{\partial z}\right| \psi_{i}(z)\right|^{2} \cos ^{2} \phi\right]\right.$ $\times \frac{(\Gamma / 2)}{\left(E_{f}-E_{i}-\hbar \omega\right)^{2}+(\Gamma / 2)^{2}}$

where, $c$ is the velocity of light, $n_{r}$ is the refractive index of material, $\hbar \omega$ is the incident photon energy, $m_{w e}^{*}$ is the electron effective mass in the well, $\Gamma$ is the broadening factor which is equal to the full width at half maximum of the absorption spectrum, $E_{i}$ and $E_{f}$ are the ground and excited state energies respectively, $\psi_{i}(z)$ and $\psi_{f}(z)$ are respectively the initial and final state wave functions. Similarly, for bound-to-continuum (B-C) transition, the absorption coefficient $\alpha_{C_{B-B}}(\hbar \omega)$ can be written as

$$
\begin{aligned}
& \alpha_{C_{B-B}}(\hbar \omega)=\frac{q^{2}}{\pi\left(m_{w e}^{*}\right)^{2}} \frac{\hbar^{2} N}{\varepsilon_{0} n_{r} c} \sqrt{\frac{\Gamma / 2}{\left(E_{f c}-E_{i}-\hbar \omega\right)^{2}+\left(\Gamma^{2} / 4\right)}} \\
& \sqrt{\frac{m_{B e}^{*}}{2\left(E_{f c}-V_{0}\right)}\left[\left|\left\langle\psi_{f c}(z)\left|\frac{\partial}{\partial z}\right| \psi_{i}(z)\right\rangle\right|^{2} \cos ^{2} \phi\right],}
\end{aligned}
$$

where, $E_{f_{c}}$ and $\psi_{f c}(z)$ are respectively the Eigen energy and wave functions of the continuum state, $m_{B e}^{*}$ is the electron effective mass in the barrier and $V_{0}$ is the effective band offset due to tilt of energy bands under bias. It may be mentioned here that the wave functions such as $\psi_{i}(z), \psi_{f}(z)$ and $\psi_{f_{c}}(z)$ can be written as

$$
\psi_{i, f, f_{c}}(z)=\left[\psi_{1}(z) \psi_{2}(z) \psi_{3}(z)\right]
$$

here, $\psi_{1}(z), \psi_{2}(z)$ and $\psi_{3}(z)$, i.e., $\psi_{r}(z)$ (where $r=1,2$ and 3 ) can be calculated from Eq. (4) [under no-bias] and Eq. (5) [under bias], respectively. But the term $k_{r}$ in Eq. (4) should be modified as $k_{r}=i k_{r}=i \sqrt{2 m_{r e}^{*}\left(V_{r}-E_{i, f}\right) / \hbar^{2}}$ for $E_{i, f}<V_{r}$ and $k_{r}=\sqrt{2 m_{r e}^{*}\left(E_{f c}-V_{r}\right) / \hbar^{2}}$ for $E_{f c}>V_{r}$. Similarly, $l_{r}$ in Eq. (5) should be modified as $l_{r}=\left(E_{i, f, f_{c}}-V_{r}\right) / q$.

Knowing absorption coefficient and hence generation of carriers, the photocurrent density in a single quantum well and hence in a multiple quantum well (MQW) structure is calculated as follows. Carrier density (only electrons here) in a single quantum well can be obtained from solution of continuity equation and rate equation. Neglecting diffusion component of current, continuity equation for a single well, say for $j^{\text {th }} \mathrm{QW}$ can be written as

$$
\frac{\partial n_{j}(z, t)}{\partial t}=g_{j}(z, t)-\frac{n_{j}-n_{0}}{\tau_{r}}+v_{e} \frac{\partial n_{j}(z, t)}{\partial z},
$$

where, $n_{0}$ and $n_{j}$ are electron densities under equilibrium and non-equilibrium conditions (for $j^{\text {th }}$ well), respectively, $\tau_{r}$ is the carrier lifetime of electrons and $v_{e}$ is the temperature dependent saturation drift velocity of electrons. Position dependent generation of carriers in the $j^{\text {th }} \mathrm{QW}, g_{j}(z, t)$ is given as

Table 1(a). Some material parameters of $\mathrm{Al}_{\mathrm{X}} \mathrm{Ga}_{1-\mathrm{x}} \mathrm{As}$ used in calculation for different values of $x$ :

\begin{tabular}{cccc}
\hline \multirow{2}{*}{ Mole fraction $(x)$} & $m_{B e}\left(\mathrm{x} m 0=9.10939 \times 10^{-31} \mathrm{in} \mathrm{Kg}\right)$ & $\mathrm{T}=300 \mathrm{~K}$ & \multicolumn{2}{c}{ Band $\mathrm{Gap}\left(E g_{1}, \mathrm{eV}\right)$} \\
\cline { 3 - 4 } 0.24 & 0.0829 & 1.7209 & 1.8061 \\
0.25 & 0.0838 & 1.7343 & 1.8195 \\
0.26 & 0.0846 & 1.7477 & 1.8329 \\
0.27 & 0.0854 & 1.7613 & 1.8464 \\
0.28 & 0.0862 & 1.7748 & 1.86 \\
\hline
\end{tabular}

Table 1(b). Some material parameters of GaAs used in calculation for different values of $T$ :

\begin{tabular}{cccc}
\hline Temperature $(T$ in $\mathrm{K})$ & $\mathrm{m}_{w e}(\mathrm{x} m 0$ in $\mathrm{Kg})$ & Band $\mathrm{Gap}\left(E g_{2}, \mathrm{eV}\right)$ & $v_{e}(\mathrm{~m} / \mathrm{s})$ \\
\hline 10 & & 1.5187 & $1.25 \times 10^{5}$ \\
77 & 0.063 & 1.5076 & $1.07 \times 10^{5}$ \\
300 & & 1.4224 & $7.2 \times 10^{4}$ \\
\hline
\end{tabular}




$$
g_{j}(z, t)=\frac{p_{i n c} \alpha}{A \hbar \omega} e^{-\alpha j L_{w}}\left[e^{\alpha\left[L_{1}+(j-1) L_{B}+j L_{w}-z\right]}\right],
$$

where, $P_{\text {inc }}$ is the incident optical power, $A$ is the area under illumination, $L_{1}$ is the distance of the nearest well from the front of the emitter as shown in Fig. 1(a) and $\alpha$ is the absorption coefficient which needs to be replaced by $\alpha_{C_{B-B}}$ or $\alpha_{C_{B-C}}$ for bound-to-bound and bound-to-continuum transition, respectively.

Now, the rate equation for $j^{\text {th }}$ quantum well can be written as

$$
\frac{d n_{\tau}(z)}{d t}=\frac{n_{j}(z)}{\tau_{c a p}}-\left(r_{e s c}+r_{r}\right) n_{\tau}(z),
$$

where, $n_{\tau}$ is the electrons captured per volume in the well, $\tau_{c a p}$ is the capture lifetime of electrons, $r_{e s c}$ is the escape rate of electrons from the well which is inverse of escape time of electrons, $\tau_{e s c}\left(\right.$ i.e., $\left.r_{e s c}=1 / \tau_{e s c}\right), r_{r}$ is the recombination rate of electrons which is inverse of $\tau_{r}$. Capture time, $\tau_{\text {cap }}$ can be considered to be equivalent to the excited carrier lifetime, $\tau_{r}$ particularly for the device of our consideration which works under electric field [19-20]. It may be mentioned here that the capture probability, $p_{c}=\tau_{\text {trans }} /\left(\tau_{\text {trans }}+\tau_{\text {cap }}\right)$ where, $\tau_{\text {trans }}$ is the transit time of carriers to traverse one QW period (i.e., $L_{w}+L_{B}$ ) and, $\tau_{\text {trans }}<<\tau_{\text {cap }}$ and hence, $p_{c}<<1$ and its value lies in the range of 0.03-0.006 for an electric field between 1 and $10 \mathrm{kV} / \mathrm{cm}$ [20]. Moreover, thick barrier is considered in this model so, the field assisted emission is assumed to be the dominating process of escape of carriers from the well. Rate of escape of electrons, $r_{e s c}$ at applied bias, $V$ can be written as [20-21]

$$
r_{e s c}(V)=r_{e s c}(0) e^{V / V_{s}},
$$

where, $r_{\text {esc }}(0)$ is the rate of emission of electrons at zero bias and $V_{S}$ is the effective potential barrier lowering which depends on the band offset, $V(z)$, Hartree potential, $V_{H}(z)$ and Eigen energy state.

The continuity equations are solved for each well with appropriate boundary conditions. The carrier distribution in a well depends on the carrier coming from the previous well, capture and escape probabilities of carriers in the well and recombination. Considering these and after a detail calculation, position dependent photogenerated electron density, $n_{j}(z)$ in the $j^{\text {th }}$ well is obtained. After averaging this density over whole width of the well, the average electron density, $n_{j}$ in the $j^{\text {th }}$ quantum well is obtained as

$$
\begin{aligned}
& n_{j}=\frac{p_{i n c} \alpha e^{-\alpha j L_{w}}}{A \hbar \omega L_{w}}\left\{\frac{1}{v_{e}\left(\alpha+\frac{1}{\tau_{r} v_{e}}\right)}\left[\frac{e^{\alpha L_{w}-1}}{\alpha}-\frac{1-e^{-L_{w} / \tau_{r} v_{e}}}{1 / \tau_{r} v_{e}}\right]\right. \\
& +\sum_{i=1}^{M-j+1} \frac{e^{-(i-1) L_{w} / \tau_{r} v_{e}}}{v_{e}\left(\alpha+\frac{1}{\tau_{r} v_{e}}\right)} \\
& \left.\times\left[\left(e^{\alpha L_{w}}-e^{-L_{w} / \tau_{r} v_{e}}\right)\left(\frac{r_{e s c}}{r_{e s c}+r_{r}}\right)^{i-1}\right] \frac{1-e^{-L_{w} / \tau_{r} v_{e}}}{1 / \tau_{r} v_{e}}\right\}
\end{aligned}
$$

where, $M$ is the number of quantum wells. The term, $r_{\text {esc }} /\left(r_{\text {esc }}+r_{r}\right)$ in the above expression is nothing but escape probability $\left(p_{e}\right)$ of electrons from the well. So, the average photocurrent density, $J$ for multiple quantum well photodetector can be written as [22]

$$
J=\frac{1}{M} \sum_{j=1}^{M} q n_{j} v_{e}
$$

Finally, the responsivity of MQW photodetector is calculated by using the following relationship

$$
R=J A / P_{\text {inc }}
$$

\section{Results and discussion}

Values of some important material parameters of AlGaAs and GaAs, used in our MATLAB based simulation, are summarized in Table 2(a) and 1(b) respectively. In addition, the thickness of the $\mathrm{Al}_{\mathrm{x}} \mathrm{Ga}_{1-\mathrm{x}} \mathrm{As}$ barrier layer $\left(L_{B}\right)$ of $30 \mathrm{~nm}$, the incident optical power $\left(P_{i n c}\right)$ is assumed to be $1 \mathrm{~mW}$ and the device area $(A)$ is considered as $200 \times 200 \mu \mathrm{m}^{2}$. Since the normal incidence is not capable to cause inter sub band transitions in $N$-type quantum well structure as per the selection rule, the light is considered to be incident at an angle $(\phi)$, $45^{\circ}$ with the growth axis $(z)$ as shown in Fig. 1(a).

To study the effect well doping on the responsivity spectra, we need to study the absorption coefficient first which requires determination of Eigen energy states and wave function of carriers. Now, the Eigen energy states and shape of the wave functions strongly depend on the position dependent Hartree potential. Moreover, the wave function of electrons in the lowest, i.e., ground Eigen energy state, in particular, greatly depends on shape of the Hartree potential, as well. The energy Eigen states, wave function and Hartree potential can be obtained by self-consistent solution of Eq. (1) and Eq. (2). But, this solution needs to include the variation of Fermi level energy, $E_{F}$ with doping concentration which is computed and plotted for different temperatures in Fig. 2. Variation, without considering the effect of

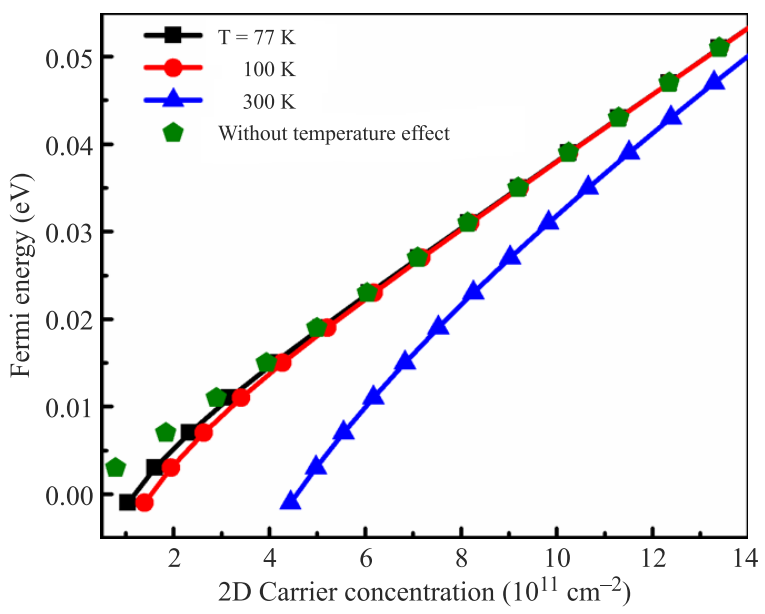

Fig. 2. (colour online) Variation of Fermi level energy as a function of $2 \mathrm{D}$ carrier concentration of a GaAs/ $\mathrm{Al}_{\mathrm{x}} \mathrm{Ga}_{1-\mathrm{x}} \mathrm{As} \mathrm{QWIP}$ for different temperature. 
temperature on Fermi Level, is also plotted in the same graph. It is clear from figure that role of temperature on $E_{F}$ is insignificant at lower range of temperatures. So, temperature independent $E_{F}$ may be considered for computation of performance parameters in the low range of temperature. However, in this work, temperature dependent $E_{F}$ is considered for all calculations. Self consistent solution of Hartree potential is obtained from Eq. (1) and Eq. (2) and is plotted, as a function of position, $z$ (growth direction) for different doping concentrations, in Fig. 3. Shape of the variation of

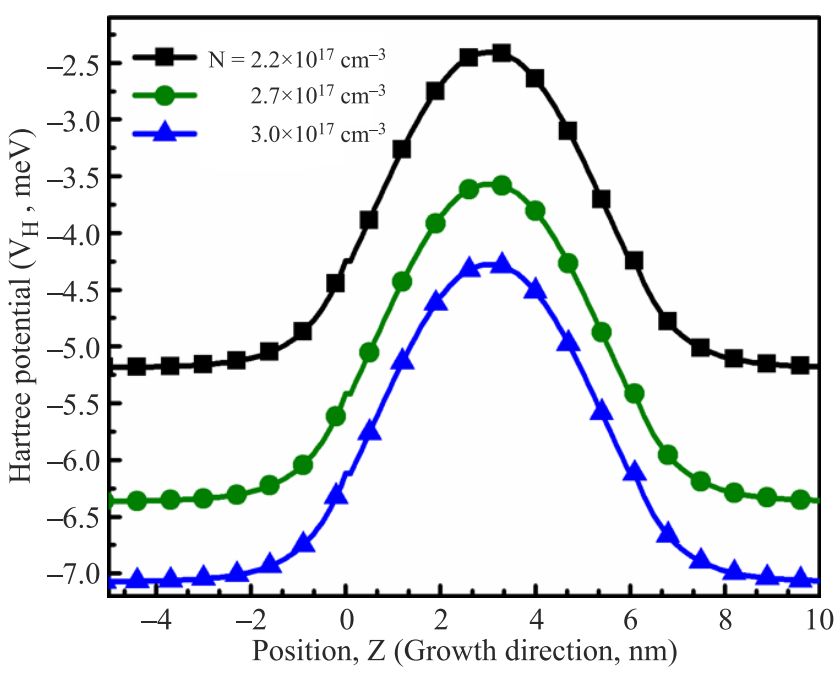

Fig. 3. (colour online) Calculated Hartree potential of a GaAs/ $\mathrm{Al}_{0.26} \mathrm{Ga}_{0.74} \mathrm{As} \mathrm{QWIP}$ with $5.8 \mathrm{~nm} \mathrm{GaAs}$ well doped with $2.2 \times 10^{17}$ $\mathrm{cm}^{-3}, 2.7 \times 10^{17} \mathrm{~cm}^{-3}$ and $3.0 \times 10^{17} \mathrm{~cm}^{-3} \mathrm{Si}$ donors at $\mathrm{T}=300 \mathrm{~K}$.

Hartree potential is parabolic in nature and it has maxima at the centre of the well. It is seen that maximum built-in potential is about $4.3 \mathrm{meV}$ at room temperature for doping density of $3.0 \times 10^{17} \mathrm{~cm}^{-3}$ in the well of width, $5.8 \mathrm{~nm}$. It is also observed that the value of Hartree potential increases with the increasing doping concentration in the well. So, the Eigen energy states, wave function and hence, absorption spectra of the device can be controlled by controlling doping concentration in the well. After obtaining Eigen energies and wave functions, absorption coefficient is determined using Eq. (6) or Eq. (7). Absorption spectra for different doping concentrations are shown in Fig. 4. For $5.2 \mathrm{~nm}$ wide well is doped with $5 \times 10^{17} \mathrm{~cm}^{-3} \mathrm{Si}$ donors, the peak absorption coefficient is obtained at wavelength of $8.57 \mu \mathrm{m}$ which is in good agreement with the available experimental data in Ref. 23 where peak absorption coefficient is obtained at $8.75 \mu \mathrm{m}$. Slight discrepancy in this result may be due to the use of a simplified $2 \times 2$ Hamiltonian method and due to the choice of conduction band discontinuity [24] because there are discrepancies in its reported values. It is seen from Fig. 4 that the peak absorption makes a blue shift with decreasing doping density in the well. Increase in doping density results in increment of Hartree potential which in turn modifies the Eigen energy states, wave function and, hence, absorption. Peak value of absorp-

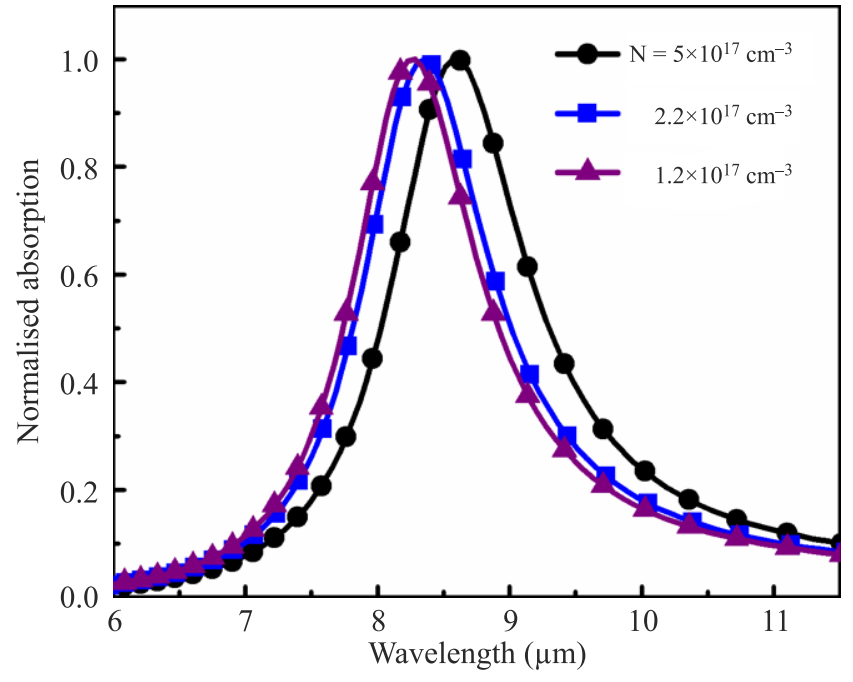

Fig. 4. (colour online) Normalized absorption $v s$. wavelength for different doping concentration of a GaAs/ $\mathrm{Al}_{0.26} \mathrm{Ga}_{0.74} \mathrm{As}$ QWIP with $5.2 \mathrm{~nm} \mathrm{GaAs}$ wide well layer at $\mathrm{T}=300 \mathrm{~K}$.

tion coefficient proportionately varies with doping concentration which can be clearly understood from Eq. (6) and Eq. (7). It is important to mention at this juncture that similar variation of normalized absorption was observed by Gunapala et al. as reported in the literature [14].

Well width also has a role on the absorption spectra because the positions of quantized energy states, as well as the number of states inside the quantum well can be varied by controlling the well width. The variation of normalized absorption as a function of wavelength for different widths of the well is plotted in Fig. 5. It is seen from figure that with increasing well width, the peak value of absorption coefficient increases and the peak absorption makes a red shift, i.e., shifts towards longer wavelengths. It is also seen that at higher values of well width, spectral width of absorption spectra becomes narrower. This is due to the fact that with

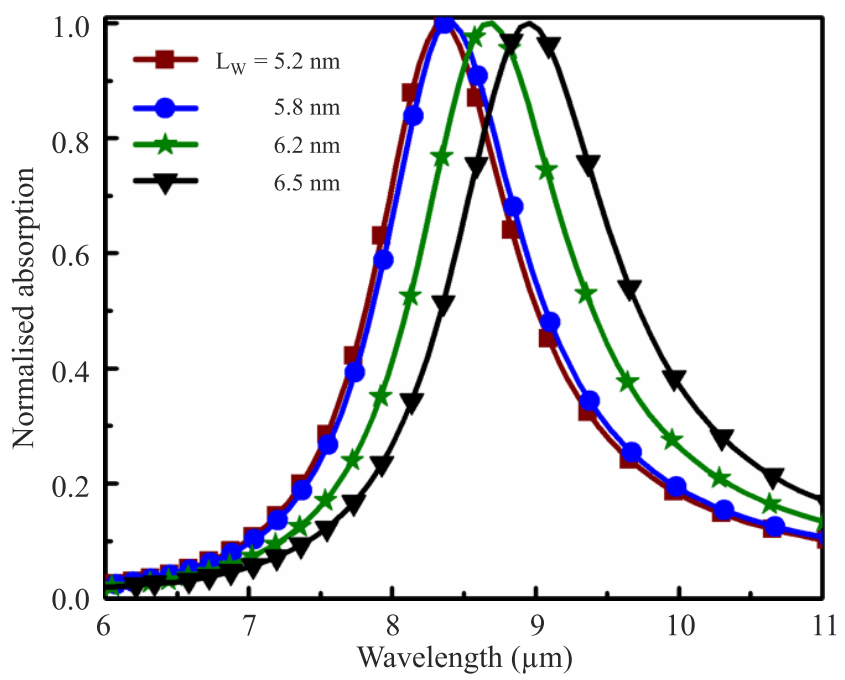

Fig. 5. (colour online) Absorption vs. wavelength of a GaAs/ $\mathrm{Al}_{0.26} \mathrm{Ga}_{0.74} \mathrm{As} \mathrm{QWIP}$ for different width of the well $\left(L_{w}\right)$ doped with $2.2 \times 10^{17} \mathrm{~cm}^{-3}$ at $\mathrm{T}=300 \mathrm{~K}$. 
increasing well width from $5.2 \mathrm{~nm}$ to $6.2 \mathrm{~nm}$, the effective band offset due to conduction band discontinuity and built-in potential changes from $0.2186 \mathrm{eV}$ to $0.2191 \mathrm{eV}$. Hence, the quantized energy states change from $E_{1}=0.0693 \mathrm{eV}$ and $E_{2}=0.2182 \mathrm{eV}$ to $E_{1}=0.0565 \mathrm{eV}$ and $E_{2}=0.1998 \mathrm{eV}$, respectively ( $E_{1}$ and $E_{2}$ are the ground and excited energy state). As a result, bound to bound transition is observed which causes the increase in absorption, as well as less broadening. Also, the number of quantized states increases with the increasing well width. Absorption can be tuned by controlling the composition of $\mathrm{Al}$ in the GaAs well. Dependence of absorption spectra on Al mole fraction $(x)$ is illustrated in Fig. 6 where $5.8 \mathrm{~nm}$ wide well is doped with

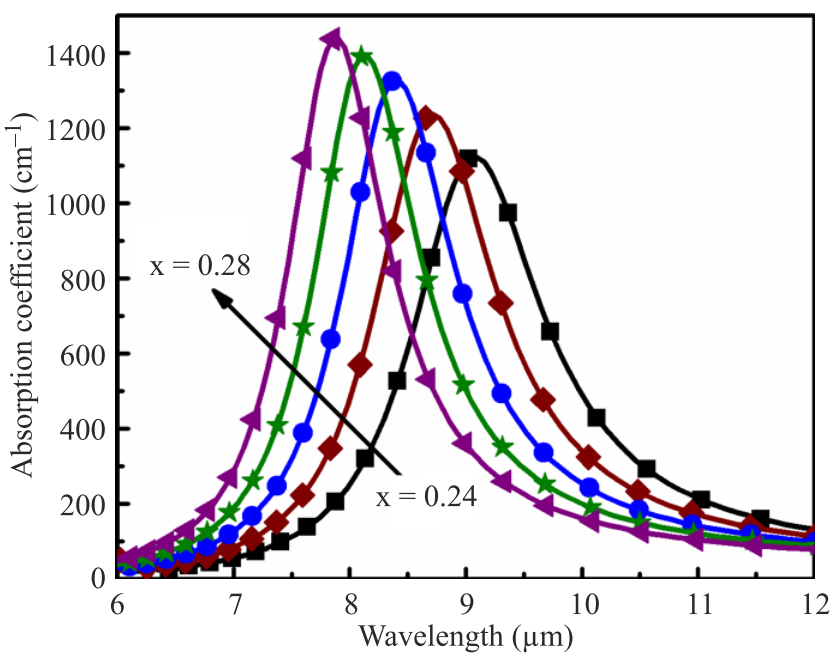

Fig. 6. (colour online) Variation of Absorption Coefficient for different values of $\mathrm{Al}$ mole fraction of a GaAs/ $\mathrm{Al}_{\mathrm{x}} \mathrm{Ga}_{1-\mathrm{x}} \mathrm{As}$ QWIP with $5.8 \mathrm{~nm}$ GaAs well doped with $2.2 \times 10^{17} \mathrm{~cm}^{-3} \mathrm{Si}$ donors at $\mathrm{T}=300 \mathrm{~K}$.

$2.2 \times 10^{17} \mathrm{~cm}^{-3} \mathrm{Si}$ donors. Conduction band discontinuity increases with $x$ so, the excited, as well as ground state shift upwards. For example, energy shifts from $E_{1}=0.0593 \mathrm{eV}$ and $E_{2}=0.1963 \mathrm{eV}$ to $E_{1}=0.0628 \mathrm{eV}$ and $E_{2}=0.2205 \mathrm{eV}$, respectively with the increase in mole fraction from $x=0.24$ to 0.28 . As a result, the peak absorption wavelength shifts towards shorter wavelength and its value also increases. Proper choice of device parameters such as well and barrier width, doping concentration and mole fraction of $\mathrm{Al}$ allow us to select the B-B and B-C transitions. However, absorption spectra for these transitions are not the same in nature. So, it is important to study the variation of absorption spectra for both of these transitions. Figure 7 represents the normalized absorption coefficient B-B and B-C transitions at $\mathrm{V}=0.75 \mathrm{~V}$. It is observed that the $\mathrm{B}-\mathrm{B}$ transition has narrower spectral broadening as compared to the B-C transition. This can be understood by following the explanation for variation of absorption with well width in Fig. 5 shown earlier. Thus, by changing well width, doping concentration and mole fraction, position of the highest energy state in the well can be set to obtain resonance condition. At this condi-

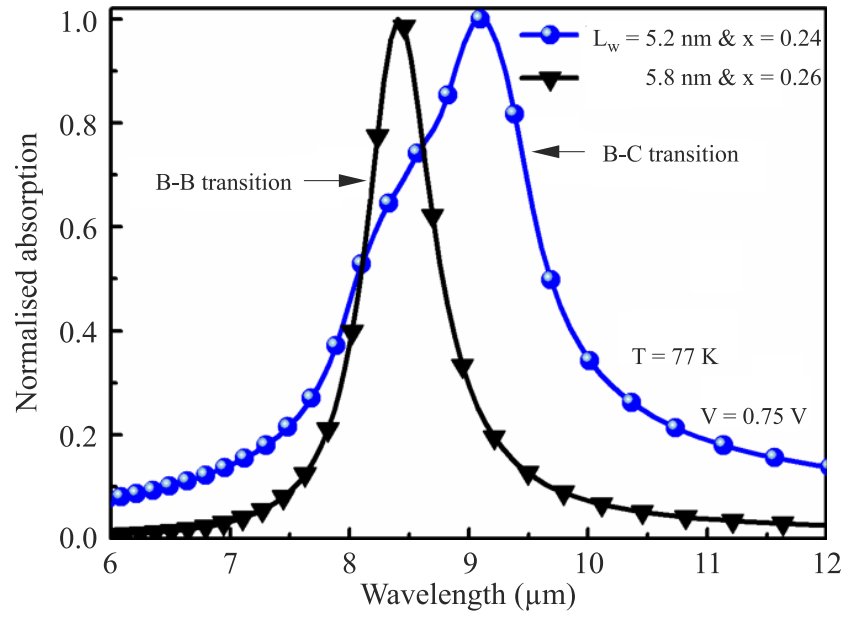

Fig. 7. (colour online) Normalized absorption based on B-B and B-C transition of a GaAs/ $/ \mathrm{Al}_{\mathrm{x}} \mathrm{Ga}_{1-\mathrm{x}} \mathrm{As}$ QWIP where GaAs well doped with $2.2 \times 10^{17} \mathrm{~cm}^{-3} \mathrm{Si}$ donors.

tion, highest energy state is almost aligned with top of the barrier so that only small bias is sufficient to obtain significant photocurrent.

To calculate photocurrent and, hence responsivity in MQW photodetector, emission process of electrons from one well is very important. In this work, we have considered a thick barrier which is almost equal or above five times thicker than well. So, the contribution of tunnelling current is negligible. Validity of this assumption can be understood from Fig. 8 where transmission coefficient for different widths of the well is shown. Very low value of transmission coefficient confirms that the contribution of tunnelling current is negligible for the structure considered in our analysis. Field induced emission of electrons is considered to be the main contribution of photocurrent and it is calculated using Eq. (14). Based on this current, responsivity is obtained

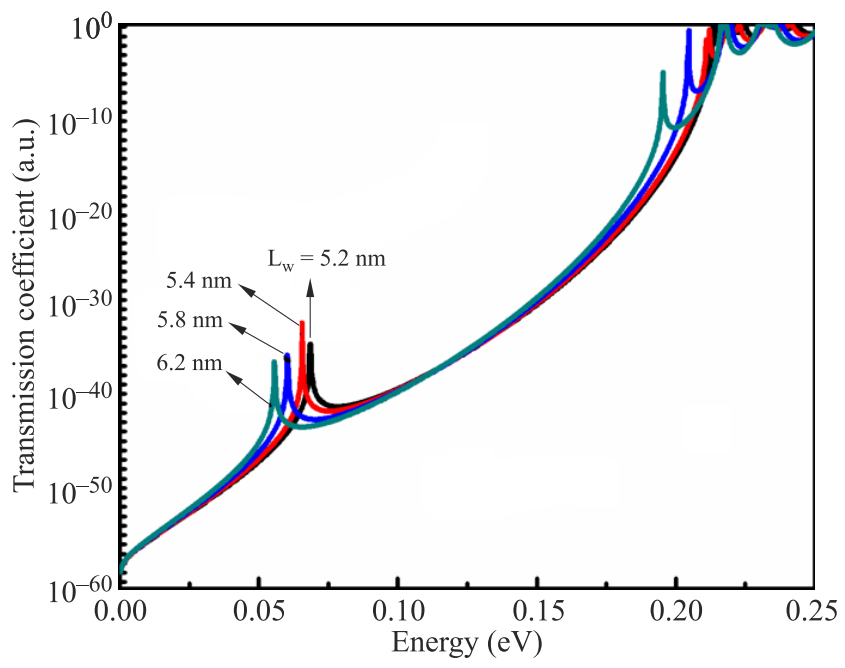

Fig. 8. (colour online) Transmission coefficient of a GaAs/ $\mathrm{Al}_{0.26} \mathrm{Ga}_{0.74}$ As double QWIP with $5.2 \mathrm{~nm}\left(L_{B} / L_{w}=5.77\right), 5.4 \mathrm{~nm}$ $\left(L_{B} / L_{w}=5.55\right), 5.8 \mathrm{~nm}\left(L_{B} / L_{w}=5.17\right)$ and $6.2 \mathrm{~nm}\left(L_{B} / L_{w}=4.84\right)$ GaAs width of the well. 


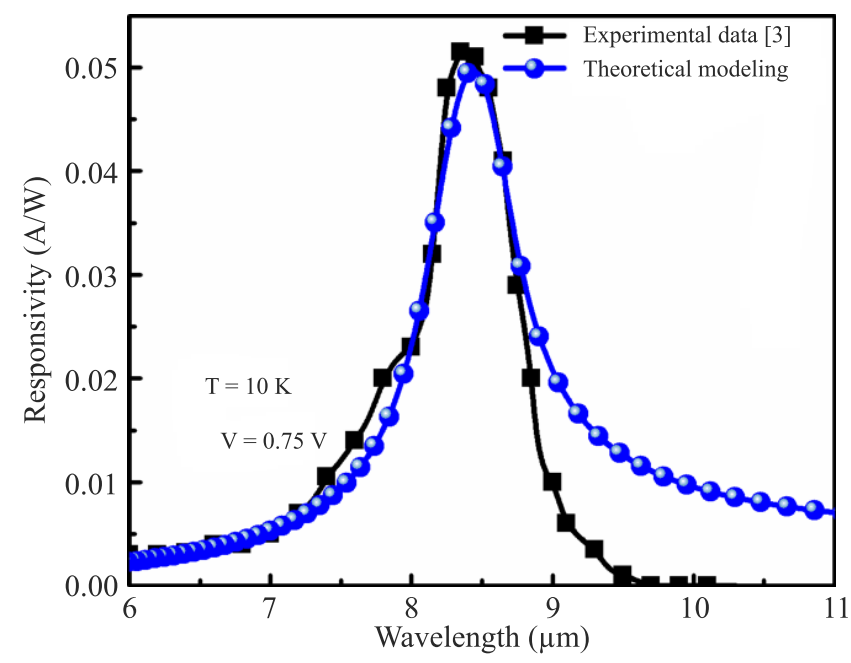

Fig. 9. (color online) Comparison of calculated responsivity spectra of a 20 periods $\mathrm{GaAs} / \mathrm{Al}_{0.26} \mathrm{Ga}_{0.74} \mathrm{As}$ MQW infrared photodetector with $5.2 \mathrm{~nm} \mathrm{GaAs}$ well doped with $5 \times 10^{17} \mathrm{~cm}^{-3} \mathrm{Si}$ donors at $\mathrm{T}=10 \mathrm{~K}$.

from Eq. (15) and its variation as a function wavelength is shown in Fig. 9. We took the occasion of verifying our model by plotting experimental values of responsivity in the same graph. Experimental values of responsivity for similar structure are extracted from literature [3]. Result shows a good agreement of our model with the experimental data which confirms the clear and unambiguous validation of this work. In this framework, it is important to note that the shape of the responsivity spectra strongly depends on the shape of absorption coefficient which, in turn, depends on spectral broadening factor, $\Gamma$ as mentioned in Eq. (7). In our simulation, small bias is assumed to be applied to the detector and, hence bound-to-continuum transition is considered in the calculation of absorption coefficient using Eq. (7). The spectral broadening parameter is chosen to be $6 \mathrm{meV}$ for bound-to-continuum transition, a reasonable value for GaAs/AlGaAs, as a fitting parameter to match the shape of the calculated result with the experimental data. Slight variation between theoretical and experimental values may be due to the following fact. Values of material parameters like electron mobility, saturation drift velocity etc. are taken from literatures other than Ref. 3 from which the experimental values of responsivity are taken since their values are not mentioned in this literature. We have used electron mobility $(\mu)$ in the range of $1000 \mathrm{~cm}^{2} \mathrm{~V}^{-1} \mathrm{~s}^{-1}$ and saturation drift velocity of $7.2 \times 10^{6} \mathrm{~cm}^{-1}$ at $T=300 \mathrm{~K}$ which are in accordance with some measured values reported in literature [5,25-27]. Zero bias escape time of electrons varies from $10^{-14} \mathrm{~s}$ to $10^{-12} \mathrm{~s}$ and it is different in the case of B-C and $\mathrm{B}-\mathrm{B}$ transition in accordance to some reported literature [20-21]. In this analysis, escape time of electrons at zero bias is taken as $1.4 \times 10^{-13} \mathrm{~s}$ and $26 \times 10^{-13} \mathrm{~s}$ respectively, for $\mathrm{B}-\mathrm{C}$ and $\mathrm{B}-\mathrm{B}$ transition. There are some discrepancies in the values of carrier lifetime $\left(\tau_{r}\right)$ as reported in different literatures $[15,20,27-28]$. However, all the reported values

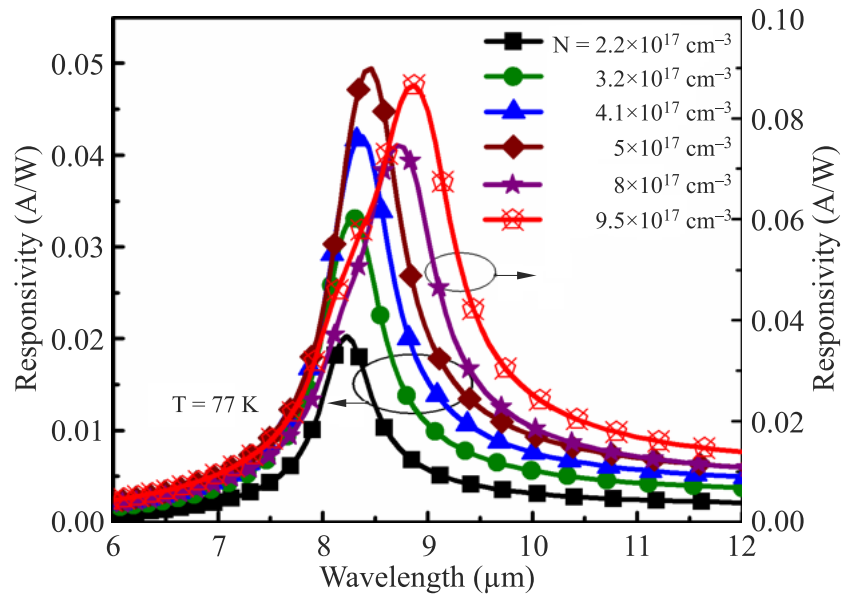

Fig. 10. (colour online) Variation of responsivity vs. wavelength of a 20 periods GaAs/ $\mathrm{Al}_{0.26} \mathrm{Ga}_{0.74} \mathrm{As} \mathrm{MQW}$ infrared photodetector with $5.2 \mathrm{~nm} \mathrm{GaAs}$ well doped with $2.2 \times 10^{17} \mathrm{~cm}^{-3}, 3.2 \times 10^{17} \mathrm{~cm}^{-3}$, $4.1 \times 10^{17} \mathrm{~cm}^{-3}, 5.0 \times 10^{17} \mathrm{~cm}^{-3}, 8.0 \times 10^{17} \mathrm{~cm}^{-3}$ and $9.5 \times 10^{17} \mathrm{~cm}^{-3} \mathrm{Si}$ donors at $\mathrm{V}=0.75 \mathrm{~V}$.

are in the order of ps and in our calculation it is taken as 6 ps.

Effect of doping on the peak responsivity in a MQW photodetector can be clearly seen from Fig. 10 where doping concentration is taken as a parameter. It is clear from figure that the peak responsivity increases with the increase in doping concentration and the peak shifts towards longer wavelength region. This is due to the variation of an absorption coefficient with doping which has already been explained earlier. Spectral width also increases with doping concentration. For better understanding of the variation of peak responsivity and wavelength with doping, these parameters are plotted as a function of doping and are shown in Fig. 11. It is interesting to observe that the peak response and wavelength varies nonlinearly with doping. In the range of

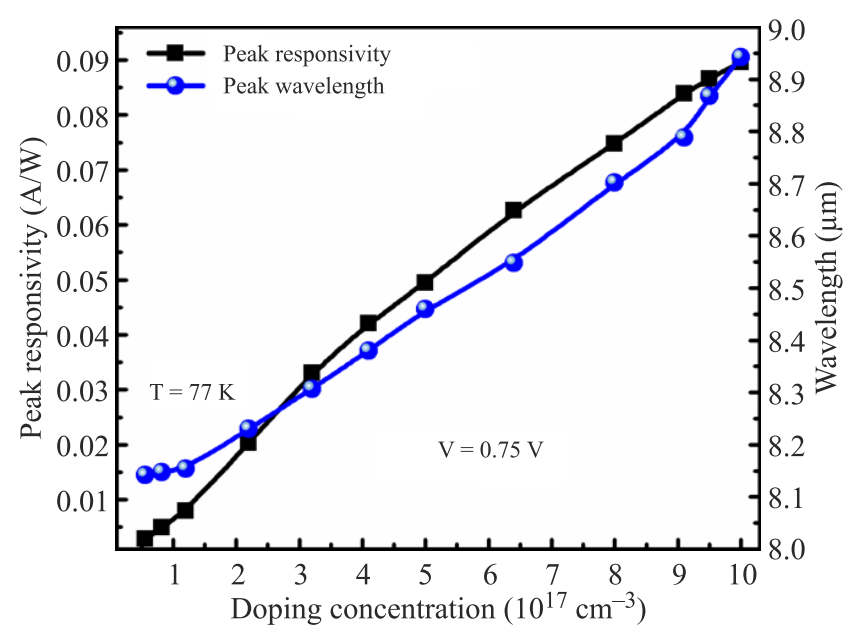

Fig. 11. (colour online) Variation of peak responsivity along with peak wavelength as a function of doping concentration of 20 periods $\mathrm{GaAs} / \mathrm{Al}_{0.26} \mathrm{Ga}_{0.74} \mathrm{As} \mathrm{MQW}$ infrared photodetector with $5.2 \mathrm{~nm}$ GaAs well width. 
$N<1.2 \times 10^{17} \mathrm{~cm}^{-3}$, effect of doping on the peak wavelength is insignificant whereas for $N>1.2 \times 10^{17} \mathrm{~cm}^{-3}$, the effect is significant. So, the desired wavelength (in infrared region) of operation can be obtained by proper choice of doping in the well. Responsivity can be enhanced also by choosing doping concentration. It is important to mention here that the well width and Al mole fraction also have similar effect on the photocurrent and, hence responsivity of the device because the absorption coefficient varies with these parameters significantly and these variations have already been shown in some previous graphs. Though the variation of responsivity with well width and $\mathrm{Al}$ mole fraction is not shown in figure, some values of responsivity, operational wavelength, spectral widths for different combinations of doping concentration, well width and $\mathrm{Al}$ mole fraction are summarized in Table 2 for quick reference.

Table 2. Some values of performance parameters of GaAs/ $\mathrm{Al}_{\mathrm{x}} \mathrm{Ga}_{1-\mathrm{x}} \mathrm{As}$ MQW IP: Bias voltage $V=0.75 \mathrm{~V}, M=20$, Peak Responsivity $R_{p}$, Peak wavelength $\lambda_{p}$, Spectral Width $\Delta \lambda$ at $T=77 \mathrm{~K}$.

\begin{tabular}{cccccc}
\hline$L_{w}(\mathrm{~nm})$ & $x$ & $\mathrm{~N}\left(\times 10^{17} \mathrm{~cm}^{-3}\right)$ & $R_{p}(\mathrm{~A} / \mathrm{W})$ & $\lambda_{p}(\mu \mathrm{m})$ & $\Delta \lambda(\mu \mathrm{m})$ \\
\hline \multirow{3}{*}{5.2} & \multirow{3}{*}{0.26} & 2.2 & 0.02 & 8.23 & 0.69 \\
& & 5.0 & 0.049 & 8.46 & 1.14 \\
& 9.5 & 0.087 & 8.87 & 1.35 \\
\hline \multirow{3}{*}{5.2} & \multirow{3}{*}{0.24} & 2.2 & 0.019 & 9.10 & 1.59 \\
& & 5.0 & 0.042 & 9.45 & 1.92 \\
& & 9.5 & 0.073 & 9.96 & 2.58 \\
\hline \multirow{3}{*}{5.8} & \multirow{3}{*}{0.26} & 2.2 & 0.074 & 8.40 & 0.72 \\
& & 5.0 & 0.164 & 8.68 & 0.76 \\
& & 9.5 & 0.185 & 8.77 & 0.88 \\
\hline
\end{tabular}

The study of the dark current is also important in this analysis since it limits the QWIP performance. Some of the reported literature such as Levine et al. [29] and others [30-34], the origin of the dark current is studied. In our analysis, the effect of doping concentration on the dark current is presented in Fig. 12. It is clearly seen that the value of dark current density increases with increasing doping concentration in the well. For a particular doping concentration, dark current increases rapidly at lower range of bias voltages but at slower rate in the high range of bias voltages. This is due to the saturation of drift velocity of electron at high bias.

\section{Conclusions}

Based on theoretical modelling, the effect of doping concentration and some device parameters on the performance of $\mathrm{GaAs} / \mathrm{Al}_{\mathrm{x}} \mathrm{Ga}_{1-\mathrm{x}} \mathrm{As} \mathrm{QWIP}$ is analyzed. Peak responsivity and spectral width increase nonlinearly with doping in the well. Also, the peak shifts towards longer wavelengths with increase in doping. Thus doping plays an important role in deciding the wavelength of operation of infrared photodetector and enhanced responsivity can be obtained by proper

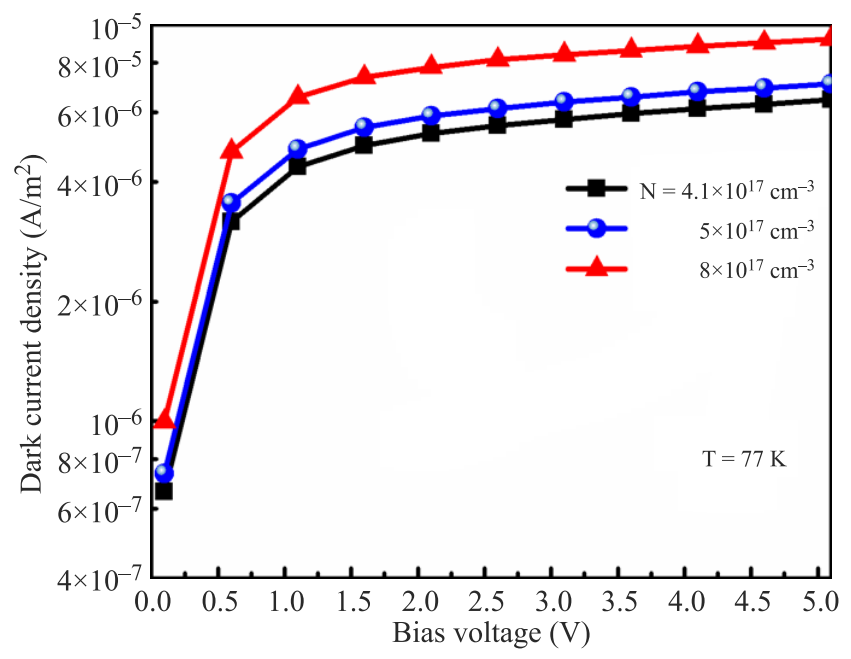

Fig. 12. (colour online) Variation of dark current vs. bias voltages for different doping concentration of a 20 periods GaAs/ $\mathrm{Al}_{0.26} \mathrm{Ga}_{0.74} \mathrm{As} \mathrm{MQW}$ infrared photodetector with $5.2 \mathrm{~nm} \mathrm{GaAs}$ well width.

choice of doping concentration. Performance of the device depends also on some device parameters and other material parameters like width of the well, mole fraction of $\mathrm{Al}$ in GaAs well, etc. With increasing well width, peak absorption coefficient increases and the peak shifts towards longer wavelength. But with increase in mole fraction of $\mathrm{Al}$ in the barrier, the peak absorption shifts towards shorter wavelength. However, absorption increases with increase in $\mathrm{Al}$ mole fraction. Responsivity of the device also varies with width of the well and $\mathrm{Al}$ mole fraction and, hence enhanced responsivity can be obtained by a proper choice of these parameters. Moreover, the dark current can be reduced by the decreasing doping concentration in the well. Thus choice of the doping concentration is one of the important parameters in design of high performance QWIP.

\section{Acknowledgements}

This work is supported in part by a grant through SAP under UGC, Govt. of India.

\section{References}

1. B.F. Levine, "Quantum-well infrared photo-detectors," $J$. Appl Phys. 74, R1 (1993).

2. T. Mei, H.Li, G. Karunasiri, W.J. Fan, D.H. Zhang, S.F. Yoon, and K.H. Yuan, "Normal incidence silicon doped p-type GaAs/AlGaAs quantum-well infrared photodetector on (111) ^ substrate," Infrared Phys. \& Tech. 50, 119-123 (2007).

3. F.D.P. Alves, J. Amorim, M. Byloos, H.C. Liu, A. Bezinger, M. Buchanan, N. Hanson, and G. Karunasiri, "Three band Quantum well infrared photodetector using interband and intersubband transitions", J. Appl. Phys. 103, 114-515 (2008).

4. V. Ryzhii, "Characteristics of quantum well infrared photodetectors", J. Appl Phys. 81, 6442 (1997). 
5. L. Thibaudeau, P. Bois, and J. Y. Duboz, "A self-consistent model for quantum well infrared photodetectors," J. Appl Phys. 79, 446 (1996).

6. B.K. Janousek, M.J. Daugherty, W.L. Bloss, M.L. Rosenbluth, M.J. O’Loughlin, H. Kanter, F.J. De Luccia, and L.E. Perry, "High-detectivity GaAs quantum well infrared detectors with peak responsivity at $8.2 \mu \mathrm{m}$ ", J. Appl. Phys. 67, 7608 (1990).

7. C.G. Bethea, B.F. Levine, V.O. Shen, R.R. Abbott, and S.J. Hseih, "10 $\mu \mathrm{m}$ GaAs/AlGaAs multiquantum well scanned array infrared imaging camera", IEEE Trans. Electron. Devices 38, 1118-1123 (1991).

8. L.J. Kozlowski, G. M. Williams, G.J. Sullivan, C.W. Farley, R.J. Andersson, J.K. Chen, D.T. Cheung, W.E. Tennant, and R.E. DeWames, "LWIR 128*128 GaAs/AlGaAs multiple quantum well hybrid focal plane array", IEEE Trans. Electron. Devices 38, 1124-1130 (1991).

9. A. Rogalski, "Infrared detectors: An overview", Infra. Phys. \& Tech. 43, 187-210 (2002).

10. S.D. Gunapala and S.V. Bandara, "Significance of the first excited state position in quantum well infrared photodetectors", Microelectronics Journal. 30, 1057-1065 (1999).

11. J. Moon, S.S. Li, and J.H. Lee, "A high performance quantum well infrared photodetector using superlattice-coupled quantum wells for long wavelength infrared detection", Infra. Phys. \& Tech. 44, 229-234 (2003).

12. V. Guériaux, A. Nedelcu, and P. Bois, "Double barrier strained quantum well infrared photodetectors for the 3-5 $\mu \mathrm{m}$ atmospheric window", J. Appl. Phys. 105, 114515 (2009).

13. N. Imam, Elias N. Glytsis, T.K. Gaylord, K.-K. Choi, P.G. Newman, and L. Detter-Hoskin, "Quantum-Well Infrared Photodetector Structure Synthesis: Methodology and Experimental Verification" J. Quant. Elec. 39, 468 (2003).

14. Y. Yang, H.C .Liu, W.Z. Shen, N. Li, W. Lu, Z.R. Wasilewski, and M. Buchanan,"Optimal Doping density for quantum well infrared photodetector performance", J. Quant. Elec. 45, 623-628 (2009).

15. S.D. Gunapala, B.F. Levine, L. Pfeiffer, and K. West, "Dependence of the performance of GaAs/AlGaAs quantum well infrared photodetectors on doping and bias", J. Appl. Phys. 69, 6517 (1991).

16. J. A. Cuesta, A. Sànchez, and F. D. Adame, "Self-consistent analysis of electric field effects on Si -doped GaAs", Semicon. Sci. Technol. 10, 1303-1309 (1995).

17. C. Jirauschek, "Accuracy of transfer matrix approaches for solving the effective mass Schrödinger equation", J. Quant. Elec. 45, 1059-1067 (2009).

18. J. Davies, The Physics of Low-Dimensional Semiconductors An Introduction, Cambridge University Press, Cambridge, 1998.

19. H. Schneider and H.C. Liu, Quantum Well Infrared Photodetectors Physics and Applications, Springer-Verlag, New York, 2007.

20. E. Rosencher, B. Vinter, F. Luc, L. Thibaudeau, P. Bois, and J. Nagle, "Emission and capture of electrons in multiquan- tum-well structures", IEEE Trans. Quant. Elec., 30, 2875 (1994).

21. B.F. Levine, A. Zussmann, S.D. Gunapala, M.T. Asom, J.M. Kuo, and W.S. Hobson, "Photoexcited escape probability, optical gain, and noise in quantum well infrared photodetectors," J. Appl. Phys. 72, 4429-4443 (1992).

22. M.K. Das and N.R. Das, "On optimum designs of a RCE $\mathrm{Si} / \mathrm{SiGe} / \mathrm{Si} \mathrm{MQW}$ photodetector for long wavelengh applications", Opt. Quant. Electron. 41, 539-549 (2009).

23. F.D. P. Alves, G. Karunasiri, N. Hanson, M. Byloos, H.C. Liu, A. Bezinger, and M. Buchanan, "NIR, MWIR and LWIR quantum well infrared photodetector using interband and intersubband transitions", Infrared Phys. \& Tech. 50, 182-186 (2007).

24. J.R Meyer and I. Vurguftman, "Band parameters for III-V compound semiconductors and their alloys", J. Appl. Phys. 89, 5815 (2001).

25. K.M.S.V. Bandara, B.F. Levine, R.E. Leibenguth, and M.T. Asom, "Optical and transport properties of single quantum well infrared photodetectors", J. Appl. Phys. 74, 1826 (1993).

26. R. Quay, C. Moglestue, V. Palankovski, and S. Selberherr, "A temperature dependent model for the saturation velocity in semiconductor materials", Proc. Material Sci. in Semicon. 3, 149-155 (2000).

27. B.F. Levine, C.G. Bethea, K.K. Choi, J. Walker, and R.J. Malik, "Bound-to-extended absorption GaAs superlattice transport infrared detectors," J. Appl. Phys. 64, 1591 (1988).

28. C.H. Yang, J.M. Carlson-Swindle, S.A. Lyon, and J.M. Worlock, "Hot-electron ralaxation in GaAs quantum wells", Phys. Rev. Lett. 55, 2359 (1985).

29. B.F. Levine, C.G. Bethea, G. Hasnain, V.O. Shen, E. Pelve, R.R. Abbott, and S.J. Hsieh, "High sensitivity low dark current $10 \mu \mathrm{m}$ GaAs quantum well infrared photodetector", Appl. Phys. Lett. 56, 851 (1990).

30. S.R. Andrews and B.A. Miller, "Experimental and theoretical studies of the performance of quantum-well infrared photodetectors", J. Appl Phys. 70, 993 (1991).

31. S.D. Gunapala, B.F. Levine, L. Pfeiffer, and K. West, "Dependence of the performance of GaAs/AlGaAs quantum well infrared photodetectors on doping and bias", J. Appl Phys. 69, 6517 (1990).

32. A. Zussman, B.F. Levine, J.M. Kuo, and J. de Jong, "Extended long-wavelength $\lambda=11-15-\mu \mathrm{m} \mathrm{GaAs} / \mathrm{Al}_{\mathrm{x}} \mathrm{Ga}_{1-\mathrm{x}} \mathrm{As}$ quantum-well infrared photodetectors", J. Appl Phys. 70, 5101 (1991).

33. M.A. Kinch and A. Yariv, "Performance limitations of GaAs/AlGaAs infrared superlattices", Appl. Phys. Lett. 55, 2093 (1989).

34. E. Pelve, F. Beltram, C.G. Bethea, B.F. Levine, V.O. Shen, S.J. Hsieh, and R.R. Abbott, "Analysis of the dark current in doped-well multiple quantum well AlGaAs infrared photodetector", J. Appl Phys. 66, 5656 (1989). 